\title{
Intelligent English Tense Collocation and Evaluation Based on Deep Reinforcement Learning
}

\author{
Yiling Ding $\mathbb{D I}^{1,2}$ and Tianhua Wang ${ }^{1}$ \\ ${ }^{1}$ Heilongjiang University, Harbin 150080, China \\ ${ }^{2}$ Harbin Normal University, Harbin 150080, China \\ Correspondence should be addressed to Yiling Ding; dingyiling@hrbnu.edu.cn
}

Received 4 January 2022; Accepted 17 January 2022; Published 8 February 2022

Academic Editor: Jianhui Lv

Copyright (C) 2022 Yiling Ding and Tianhua Wang. This is an open access article distributed under the Creative Commons Attribution License, which permits unrestricted use, distribution, and reproduction in any medium, provided the original work is properly cited.

\begin{abstract}
The representation of time in sentences is the key problem for tense collocation. Based on the relationship expression among regions in Allen's interval algebra theory, we propose a vector representation method, i.e., relationship vector, and several operations are defined based on the relationship vector for temporal reasoning in this work. This method transforms the original matrix representation into vector representation, which reduces the amount of computation of temporal reasoning. In addition, we propose a temporal classification and collocation method based on deep learning and deep reinforcement learning. This method uses a bidirectional cyclic neural network and a convolutional neural network for text expression and achieves temporal word classification and temporal collocation based on the deep reinforcement learning model. In the experiments, the proposed method obtains the average accuracy of $92.17 \%$ in five datasets, i.e., MPQA, CR, MR, Subj, and TREC, which proves its effectiveness in tense collocation.
\end{abstract}

\section{Introduction}

Mathematical proof and simulation are the important foundation of modern science and technology, and the development of artificial intelligence (AI) is also based on strong mathematical theories [1]. Symbolization and formulation of human mind on computer have become an important topic in the research of AI. Establishing a unified theoretical system of knowledge-based human intelligence and machine intelligence is the mainstream idea of AI, and the key problem is knowledge representation, knowledge reasoning, and the application of knowledge. In recent years, with the application and development of AI and big data technology, deep learning (DL) technology has made breakthroughs in many fields, such as image recognition, speech recognition, and natural language processing (NLP) [2]. Among them, semantic analysis technology for NLP plays an important role in studying human communication mode. It affects not only the development of AI, but also the mode of human-computer interaction [3]. Using AI semantic analysis technology to analyze the language materials can significantly improve the analysis efficiency of user research results and even dig out important information that is difficult to find by artificial analysis. NLP is a technology that uses computer as a tool to process the information of human unique natural language $[4,5]$. Semantic analysis of language materials is the basis of NLP involving linguistics, computational linguistics, machine learning, cognitive language, and other disciplines. The goal of semantic analysis is to realize the automatic semantic analysis of vocabulary, sentences, and chapters and understand the meaning of language text expression by establishing effective computational analysis models. Among them, the semantic analysis of words is the basis, and the commonly used knowledge expression methods include semantic field, semantic network, concept map, and ontology [6].

There are a lot of emotions and ideas in natural language that cannot be described by simple logical relations. It is difficult to achieve because of the complexity of recognizing and understanding natural languages by computer. From the 
development history of NLP, relevant research can be divided into two categories: symbolic approaches and stochastic approaches. The symbolic approaches adopt the analysis method based on linguistic definition rules focusing on the reasoning model and the logical language understanding model, which is called rationalism. The stochastic approaches mainly study random and statistical algorithms to identify and calculate large-scale corpus [7]. Although the symbolic approaches can complete the basic analysis of a single sentence, the understanding of complex sentence patterns and even the analysis of the whole paragraph or chapter are not of high quality. The statistical method contained in the stochastic approaches provides a new idea for the research method of natural language understanding, but the early effect is not satisfactory until the emergence of DL technology in recent years. Relying on statistical methods and DL algorithms, machines can learn from big data and obtain the rule of language usage, which is greatly improved in efficiency and accuracy compared with traditional methods. In particular, in the application of Chinese semantic analysis, the effect improvement is particularly prominent. It uses the ability of automatic feature extraction in the DL model to replace the manual feature construction process in traditional machine learning, which greatly improves the effectiveness of the feature model. From the historical evolution of NLP, we can see that semantic analysis technology has been developed greatly with the intervention of machine learning methods represented by DL. Through the study of literature, it is found that many scholars have studied the application methods of intelligent semantic recognition technology, and their methods and analysis steps are also different according to different application purposes. Allen [8] proposes a temporal reasoning system based on continuous interval representation of time information. It can easily represent the temporal relationship between any two-time intervals, which have strong expression ability and are easy to understand. It is widely used in natural language understanding and other fields. However, in the reasoning operation of interval algebraic representation, repeated table searching is required, which is very time-consuming. It is very impractical for the system with high real-time requirements. Therefore, it is proposed to use interval matrix to represent temporal intervals and convert the traditional table searching into matrix operation, which is very fast. Its effect is very excellent when the real-time requirements are relatively quotient. However, the temporal relationship expressed by matrix is not intuitive.

The traditional text representation methods include word bag model and vector space model. The word bag model uses unique heat coding to represent each word as a vector composed of 0 and 1 . This method is simple and intuitive, but does not consider word order and semantic information. The vector space model treats text as points in vector space. The term frequency-inverse document frequency (TF-IDF) is commonly used to represent the importance of words and construct text feature representation. However, with the rapid increase in text data on the Internet, traditional text representation methods have some disadvantages, such as high dimension, sparse features, and loss of word order. In this case, many scholars begin to use DL, and there are three types of models for text representation and classification using neural networks [9]. The first type is the serialization model, such as convolutional neural network (CNN), recurrent neural network (RNN), and longterm and short-term memory network (LSTM). They record the text serialization information for training and carry out the text classification task after obtaining the feature representation. The second type is based on attention mechanism. The third category is structured text representation and classification model, such as recursive automatic encoder [10] and short-term and long-term memory network with tree structure. They use the predefined parse tree to divide the text hierarchy and then represent and classify the text. These text representation and classification models based on deep neural networks have been verified on public datasets and have achieved good text representation and classification performance. When constructing text features, traditional text representation methods and classification models based on deep learning often delete some useless words by means of stopping vocabulary or using parsing tree to divide text structure. They cannot delete words irrelevant to classification tasks according to different texts. Parsing trees are also constructed manually, which is time-consuming and laborious. Because reinforcement learning (RL) has the ability of autonomous learning with the rapid improvement of computing performance in recent years, the combination of DL and RL for task decision-making has been widely used. Deep reinforcement learning (DRL) has the advantage of learning knowledge by interacting with the environment [11-14]. Many scholars have applied DRL to text representation and classification tasks and achieved good results. On the basis of DRL, the text representation and classification model can independently learn words related to text classification tasks, divide sentence phrase structures, and make text feature tables. It is worth studying to show and improve the performance of text classification.

To realize the accurate collocation of English sentence tenses, we propose a word-level text representation and classification model brand model based on DRL in this work. This method extracts words and divides phrase structures related to text classification tasks from main learning and optimizes text representation and classification tasks for words and phrases, respectively. Then, the Allen interval algebra is used to represent the temporal relationship, and it is applied to the path consistency algorithm of the network graph composed of a group of temporal intervals and the constraint relationship between them. In the method, the temporal constraint consistency of the subnetwork graph composed of three adjacent nodes is used as the preprocessing technology. Furthermore, the calculation result matrix is transformed into a temporal relationship representation, and one or two eigenvalues in the atomic matrix are used to determine its corresponding temporal relationship. Thus, while improving computational efficiency, we can visually represent and understand the temporal relationship and then make interval algebra obtain better representation and reasoning knowledge in our model. The 
experimental results show that the accuracy of tense collocation in English sentences can reach 92.17\%.

The rest of this study is organized as follows. Section 2 presents the related work of tense collocation methods. Section 3 presents the detailed design on tense collocation method based on DRL. Experimental results and discussion are reported in Section 4. Finally, the conclusion of this study is in Section 5.

\section{Related Work}

Focusing on the text representation and classification model of sentences and documents, Zhang et al. [15] proposed a character-level convolutional neural network (char-CNN) model, which attempted to represent and classify text by constructing alphabet and using one-hot coding and CNN. Kim [16] proposed a word-CNN model that achieves word vector representation of static and non-static channel and English text classification. Mikolov et al. [17] proposed a language model using a cyclic neural network to study text representation and classification. Based on the work, Lai et al. [18] introduced a two-way structure to capture context information and retain a wide range of word sequence information combined with cyclic and CNN models to achieve better text representation and classification results. Tang et al. [19] proposed a gate-based cyclic neural network, which analyzed document-level content through a two-way gated recurrent neural network, and used forward and backpropagation to obtain context feature representation to improve the classification effect.

The model of text feature representation and classification using DL has achieved good performance. However, in the task of text representation and classification, CNN, RNN, and other models do not consider the text hierarchy information. For structured DL models, such as tree LSTM, the predefined parsing tree is used for text structured processing, and the parsing tree is constructed by relying on a large amount of manpower and expert experience, which requires a lot of time and energy. Therefore, some scholars began to explore whether there is an interactive way that can make the text representation and classification model extract the vocabulary information related to the text classification task through autonomous learning, divide the text into phrase structures, and improve the accuracy of text feature representation and the performance of text classification. In this demand, DRL has entered the vision of many scholars. RL belongs to the branch of machine learning, and many classical algorithms have been proposed in the early stage, such as Q-learning algorithm and policy gradient algorithm. With the in-depth application of deep neural network, many scholars began to try to combine DL and RL in various fields to solve complex perceptual decision-making tasks. In view of the successful application of DRL in the image field, this method has been introduced in the text field. Yu et al. [20] used the strategic algorithm of DRL in generative adversarial networks (GANs), which provided an idea for solving the task of serializing text.

Temporal reasoning includes the formalization of time concept and provides tools for the representation and reasoning of temporal knowledge. The research of temporal reasoning originated in the late 1950s and early 1960s and initially appeared in the form of temporal logic. In the 1980s, the research on temporal logic and reasoning was very active and had never been interrupted. A logical method is one of the main methods to study temporal reasoning. The research on temporal reasoning is mainly reflected in reliability, completeness, expression ability, decidability, and computational complexity. The research of spatial logic could be traced back to the 1950s, but it had not attracted great interest of researchers in AI, spatial database, image processing, and other fields until nearly two decades, mainly including topological spatial logic, metric spatial logic, directional relationship logic, and spatial logic integrating topology and measurement. In 2002, Bennett and Cohn [21] studied the multimodal system for spatiotemporal reasoning and proposed a multidimensional modal logic for representing spatiotemporal information. In 2006, Kudinoy et al. [22] added a difference operator based on logic $S 4$ in topological space to enhance the expression ability of logic and proved its logical completeness after adding modal operator. The azimuth relation is an important spatial relation in spatial reasoning. The topological relation between spatial objects under topological transformation remains unchanged, but the azimuth relation may change. The logic complexity based on the azimuth relation is low, which is very effective for the formal representation of natural space, and has enough ability to represent meaningful spatial knowledge. Therefore, modal logic based on the azimuth relationship can better represent knowledge in natural space.

Different from the previous work, this study proposed the DRL-based method to realize the accurate collocation of English sentence tenses. The proposed method shows more effective than baselines in tense collocation.

\section{The Detailed Methodology}

The representation of tenses is an important basis for the accurate collocation of tenses in text generation. In this work, we propose a temporal vector representation, i.e., relationship vector, based on the relationship expression method between regions in Allen's interval algebra theory. Then, we design an intelligent English tense collocation method, in which several operations are defined, including relationship vector and text representation. This section will describe the details of the algorithm.

3.1. Vector Representation of Temporal Relations. The Allen interval algebra defines 13 basic interval relations, and their meanings are shown in Figure 1, where $x$ and $y$ represent continuous temporal intervals, $x, y \in[0, T]$, and Trepresents sufficiently large time values. Among the 13 relationships, 6 pairs of relationships are mutually inverse, and the other equal relationships are mutually inverse with itself. Each basic interval relation describes the definite relationship between two temporal intervals. To describe the uncertain temporal relationship, it needs to be represented by a group 


\begin{tabular}{lcccc}
\hline Relation & Symbol & Inverse & bi \\
x before y & b & mining \\
x meets y & m & oi & si \\
x overlaps y & o & dirts y & s & fi \\
x during y & d & eq \\
x equals y & eq & f
\end{tabular}

Figure 1: 13 relations of basic interval.

of basic interval relations, which are called general interval relations.

The temporal relationship represented by matrix has some disadvantages, such as less intuition and high computational complexity. To express the temporal relationship and calculation more simply, we consider using the left and right endpoints of the interval to characterize the relationship between the endpoints of the interval. Let $[0, T]$ be a time interval taken from the time axis, and $T$ is large enough, and the interval to be investigated is always its subinterval. $I=\left[I^{-}, I^{+}\right]\left(0 \leq \Gamma \leq I^{+}>T\right)$ is one of its subintervals, i.e., $I \subseteq[0, T] . I^{L}=\left[0, I^{-}\right]$is the left outer point interval of $I, I^{-}$is the left endpoint of $I, I^{i}=\left(I^{-}, I^{+}\right)$is the inner interval of $I, I^{R}=\left[I^{-}, T\right]$ is the right outer point interval of $I$, and $I^{+}$is the right endpoint of $I .\left(I^{L}, \Gamma, I^{i}, I^{+}, I^{R}\right)$ elements are called interval $I$. Therefore, any time interval I can be written as follows:

$$
I^{L} \cup I^{-} \cup I^{i} \cup I^{+} \cup I^{R}=[0, T] .
$$

We set up $\alpha$ and $\beta$ as two subintervals of interval $[0, T]$ and define $\Delta_{\alpha \beta}^{L L}, \Delta_{\alpha \beta}^{R L}, \Delta_{\alpha \beta}^{L R}$, and $\Delta_{\alpha \beta}^{R R}$ as follows:

$$
\left\{\begin{array}{l}
\Delta_{\alpha \beta}^{L L}=\alpha^{-}-\beta^{-}, \\
\Delta_{\alpha \beta}^{R L}=\alpha^{+}-\beta^{-}, \\
\Delta_{\alpha \beta}^{L R}=\alpha^{-}-\beta^{+}, \\
\Delta_{\alpha \beta}^{R R}=\alpha^{+}-\beta^{+},
\end{array}\right.
$$

where $\alpha^{-}, \alpha^{+}, \beta^{-}$, and $\beta^{+}$are the left and right endpoints of $\alpha$ and $\beta$, respectively.

Definition 1. Setting up $\alpha$ and $\beta$ as two temporal intervals and vector $v=\left(\Delta_{\alpha \beta}^{L L}, \Delta_{\alpha \beta}^{R L}, \Delta_{\alpha \beta}^{L R}, \Delta_{\alpha \beta}^{R R}\right)$ is called basic relationship vector, in which each element is $-1,0$, or 1 . If $\Delta_{\alpha \beta}^{L L}>0, \Delta_{\alpha \beta}^{L L}=1$; if $\Delta_{\alpha \beta}^{L L}=0, \Delta_{\alpha \beta}^{L L}=0$; and if $\Delta_{\alpha \beta}^{L L}<0, \Delta_{\alpha \beta}^{L L}=-1$. $\Delta_{\alpha \beta}^{R L}, \Delta_{\alpha \beta}^{L R}$, and $\Delta_{\alpha \beta}^{R R}$ have similar value rules.

The purpose of Definition 1 is to express the basic relationship between intervals through the relationship between the left and right endpoints of intervals. Each vector obtained according to Definition 1 corresponds to a basic relationship, and thus, the basic interval relation of the Allen interval algebra can be expressed by vector, which is called the basic relationship vector. The 13 basic relationship vectors of the Allen interval algebra are listed as follows:

(1) $\alpha$ before $\beta$ can be denoted as $V_{b}=[-1,-1,-1,-1]$

(2) $\alpha$ after $\beta$ can be denoted as $V_{b}=[1,1,1,1]$

(3) $\alpha$ meets $\beta$ can be denoted as $V_{b}=[-1,0,-1,-1]$

(4) $\alpha$ met by $\beta$ can be denoted as $V_{b}=[1,1,0,1]$

(5) $\alpha$ overlaps $\beta$ can be denoted as $V_{b}=[-1,1,-1,-1]$

(6) $\alpha$ overlapped by $\beta$ can be denoted as $V_{b}=[1,1,-1$, 1]

(7) $\alpha$ finishes $\beta$ can be denoted as $V_{b}=[1,1,-1,0]$

(8) $\alpha$ finished by $\beta$ can be denoted as $V_{b}=[-1,1,-1,0]$

(9) $\alpha$ during $\beta$ can be denoted as $V_{b}=[1,1,-1,-1]$

(10) $\alpha$ includes $\beta$ can be denoted as $V_{b}=[-1,1,-1,1]$

(11) $\alpha$ starts $\beta$ can be denoted as $V_{b}=[0,1,-1,-1]$

(12) $\alpha$ started by $\beta$ can be denoted as $V_{b}=[0,1,-1,1]$

(13) $\alpha$ equals $\beta$ can be denoted as $V_{b}=[0,1,-1,0]$

In the vector representation of relationship, the rules of compound operation and transfer operation of relationship are defined as follows.

Definition 2. Compound operation of relationship (V). If two intervals $x$ and $y$ satisfy the relationship set $P=\left(p_{1}, p_{2}\right.$, $\left.\ldots, p_{k}\right), 1 \leq k \leq 13$, and $V_{j}(j=1,2, \ldots, k)$ is the atomic relationship vector corresponding to each atomic relation, then the temporal relationship vector $V_{p}$ between $x$ and $y$ can be linearly expressed by $V_{j}(j=1,2, \ldots, k)$ as follows:

$$
V_{p}=\sum_{j=1}^{k} V_{j}=[1,1, \ldots]_{1 \times k}\left[\begin{array}{c}
V_{1} \\
V_{2} \\
\vdots \\
V_{k}
\end{array}\right] .
$$

It can be known from Definition 1 that we should obtain the relationship between the left and right endpoints of $\alpha$ and $\gamma$.

Thus, the relationship between the left endpoints of $\alpha$ and $\gamma$ can be obtained from the left endpoint of $\beta$. The relationship between the right endpoint of $\alpha$ and the left endpoint of $\gamma$ can be obtained from the right endpoint of $\beta$. The relationship between the left endpoint of $\alpha$ and the right endpoint of $\gamma$ can be obtained from the left endpoint of $\beta$. The relationship between the left endpoint of $\alpha$ and the right endpoint of $\gamma$ can be obtained from the right endpoint of $\beta$. From point algebra, the relationship between points $\{<,>,=\}$ has the following characteristics:

(1) Transitivity, if the point $a<(>,=) b$ and $b<(>,=) c$, then $a<(>,=) c$

(2) Retention, if the point $a<(>) b$ and $b=c$, then $a<(>)$ c. If $a=b$ and $b<(>) c$, then $a<(>) c$ 
(3) Uncertainty, if the point $a<(>) b$ and $b>(<) c$, then the relationship between $a$ and $b$ is uncertain, which can be denoted as $a(<,>,=) b$

From the above discussion, the transfer operation of the defined relationship is as follows.

Definition 3. Relation transfer operation $(\bullet)$. Setting time interval $\alpha, \beta$, and $r_{1}, r_{2} \in U$, and if $\alpha\left\{r_{1}\right\} \beta, \beta\left\{r_{2}\right\} \gamma$, then $V_{r 1}$ and $V_{r 2}$ are their corresponding relationship vectors, and the relationship vector of $\alpha$ between $\gamma$ can be obtained by $V_{r 1}$ and $V_{r 2}$ with operation: $V_{r 1 r 2}=V_{r 1} \bullet V_{r 2}$, where $V_{r i} \mid i=1,2,3$, and 4 are the components of the relationship vector, and the value rules are as follows:

$$
V_{r 1 r 2}^{3}= \begin{cases}1, & \begin{array}{l}
\text { if both of } V_{r 1}^{3} \text { and } V_{r 2}^{4} \text { are } 0, \\
\text { or one of them is } 0 \text { and the other one is } 1, \\
\text { if both of } V_{r 1}^{3} \text { and } V_{r 2}^{4} \text { are } 1, \text { or one of } \\
\text { them is } 0 \text { and the other one is }-1,
\end{array} \\
0, & \text { if both of } V_{r 1}^{3} \text { and } V_{r 2}^{4} \text { are } 0, \\
\{-1,0,1\}, \begin{array}{l}
\text { if one of } V_{r 1}^{3} \text { and } V_{r 2}^{4} \text { is } 1 \text { and } \\
\text { the other oneis }-1,
\end{array}\end{cases}
$$

$$
\begin{aligned}
& \int 1, \quad \text { if both of } V_{r 1}^{4} \text { and } V_{r 2}^{2} \text { are } 0 \text {, or one of }
\end{aligned}
$$

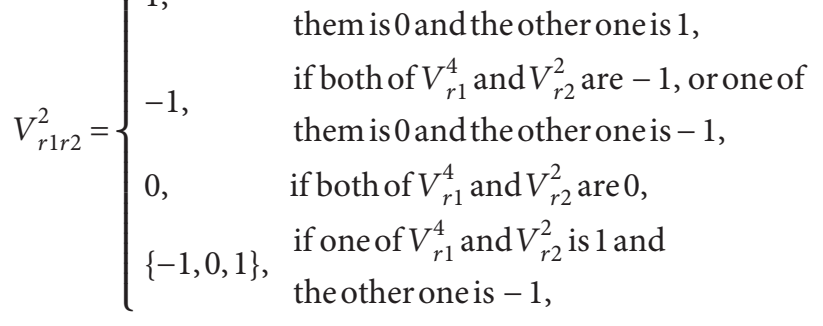

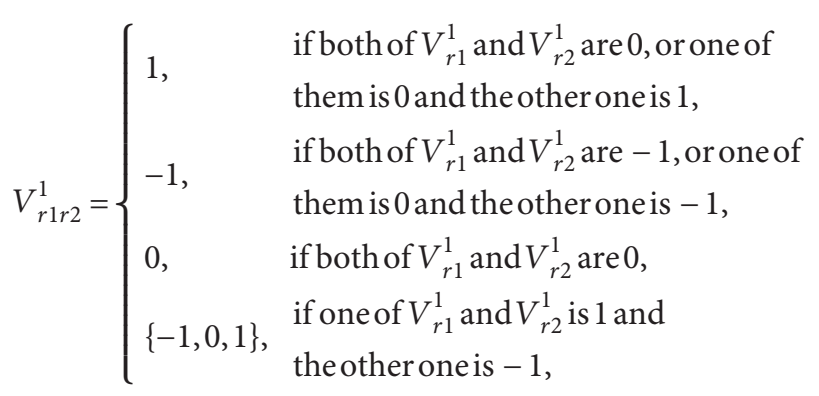

$$
V_{r 1 r 2}^{4}= \begin{cases}1, & \begin{array}{l}
\text { if both of } V_{r 1}^{4} \text { and } V_{r 2}^{4} \text { are } 0, \text { or one of } \\
\text { them is } 0 \text { and the other one is } 1,
\end{array} \\
-1, & \begin{array}{l}
\text { if both of } V_{r 1}^{4} \text { and } V_{r 2}^{4} \text { are }-1, \text { or one of } \\
\text { them is } 0 \text { and the other one is }-1, \\
\text { if both of } V_{r 1}^{4} \text { and } V_{r 2}^{4} \text { are } 0,
\end{array} \\
\{-1,0,1\}, \begin{array}{l}
\text { if one of } V_{r 1}^{4} \text { and } V_{r 2}^{4} \text { is } 1 \text { and } \\
\text { the other one is }-1,
\end{array}\end{cases}
$$

The relationship between $r_{1}$ and $r_{2}$ in transfer operation can be generalized to relational subsets $I_{1}$ and $I_{2}$.

Definition 4. Transitive operation of relation subset $(\odot)$. Setting time interval $\alpha, \beta$, and $\gamma$, and relational subsets $I_{1}$ and $I_{2} \subseteq \mathrm{U}$, if $\alpha\left\{I_{1}\right\} \beta, \beta\left\{I_{2}\right\} \gamma, V_{r 1}$, and $V_{r 2}$ are their relationship vectors, then the relationship vector $\alpha$ between $\gamma$ can be calculated by $V_{r 1}$ and $V_{r 2}$ with the operation $\odot$ as follows:

$$
\begin{aligned}
V_{I_{1} I_{2}} & =V_{I_{1}}^{T} \odot V_{I_{2}} \\
& =\left[\begin{array}{c}
V_{r 1} \\
V_{r 2} \\
\vdots \\
V_{r m}
\end{array}\right]\left[V_{R 1} V_{R 2} \ldots V_{R N}\right],
\end{aligned}
$$

where $r_{1}, \ldots, r_{m} \in I_{1}, R_{1}, \ldots, R_{n} \in I_{2}$, and $V_{r 1}$ and $V_{R 1}$ are their corresponding relationship vectors.

\subsection{Word-Level Text Representation with Tense Collocation} Based on DRL. Considering the slow training speed of strategic network in DRL, it is necessary to pre-train the original DL model without strategic network to accelerate and stabilize strategic network training and verify its effect on word extraction related to classification tasks in text. Therefore, before designing the overall word-level text representation and classification model based on DRL, we determine a DL model with excellent text representation and classification performance as the basic model, which is selected based on bidirectional recurrent neural network and convolution neural network (BRCNN). This model consists of two parts, including bidirectional cyclic neural network and CNN, in which the bidirectional cyclic neural network is used to extract the forward and reverse word order information of the text, and $\mathrm{CNN}$ is used to obtain the feature representation of the text.

3.2.1. Input of the Model. We focus on the text representation and classification of sentences in this model, and the network input is the word vector matrix $\left(x_{1}, x_{2}, \ldots, x_{N}\right)^{T} \in R^{N \times D}$ corresponding to the $i$ th word in the sentence, i.e., $x_{i} \in R^{D}, D$ represents the dimension of the word vector, and $N$ represents the number of words in the sentence.

3.2.2. Bidirectional Cyclic Neural Network. After the input vector $\left(x_{1}, x_{2}, \ldots, x_{N}\right)^{T}$ passes through the bidirectional cyclic neural network, the forward word order information and backward word order information can be obtained, which are $\left(\vec{h}_{1}, \vec{h}_{2}, \ldots, \vec{h}_{N}\right)$ and $\left(\overleftarrow{h}_{1}, \overleftarrow{h}_{2}, \ldots, \overleftarrow{h}_{N}\right)$, respectively. Attention mechanism contains two groups of weight factors, i.e., forward weighting factors $\left(\vec{\alpha}_{1} \cdot \vec{h}_{1}, \vec{\alpha}_{2}\right.$. $\vec{h}_{2}, \ldots, \quad \vec{\alpha}_{N} \cdot \vec{h}_{N}$ ) and backward weighting factor $\left(\vec{\alpha}_{1} \cdot \vec{h}_{1}, \overleftarrow{\alpha}_{t} \cdot \overleftarrow{h}_{2}, \ldots, \vec{\alpha}_{N} \cdot \vec{h}_{N}\right)$, respectively. The word order information vectors output by the cyclic neural network at each forward and backward time are weighted 
and cascaded to obtain $\vec{O}$ and $\overleftarrow{O}$. We cascade $\vec{O}$ and $\overleftarrow{O}$ to obtain the context vector $O_{R N N}$, which is shown as follows:

$$
O_{R N N}=[\vec{O}, \stackrel{\leftarrow}{O}] \text {, }
$$

where $O_{R N N} \in R^{N \times 2 K}$, in which $K$ is the number of hidden layer neurons.

3.2.3. CNN Model. The input of CNN is the output $O_{R N N}$ of bidirectional cyclic neural network. In BRCNN, the size of convolution kernel $f$ is $S \times 2 K$, where $S$ is the window size of convolution kernel corresponding to the number of hidden states $O_{R N N}$ in each convolution. $2 \mathrm{~K}$ is the convolution kernel width, which is consistent with the vector dimension in $O_{R N N}$. The output $O_{R N N}$ of bidirectional cyclic neural network can obtain the output vector after convolution operation of convolution kernel. The BRCNN model designed in this work uses three different convolution kernels $f_{1}, f_{2}$, and $f_{3}$, and their sizes are $S_{1} \times 2 K, S_{2} \times 2 K$, and $S_{3} \times 2 K$, respectively. After the output of the bidirectional cyclic neural network, i.e., $O_{R N N}$, passes through three convolution kernels $f_{1}, f_{2}$, and $f_{3}, O_{1} \in R^{N \_}{ }^{S 1+1}, O_{2} \in R^{N \_S 2+1}$, and $O_{3} \in R^{N}{ }^{N}{ }^{3+1}$ can be obtained as shown in Figure 2.

We assume that the number of convolution kernels in each type is $M$, and $O_{1} \in R^{M \times\left(N_{-} S 1+1\right)}, O_{2} \in R^{M \times\left(N_{-} S 2+1\right)}$, and $\mathrm{O}_{3} \in R^{M \times\left(N_{-} S 3+1\right)}$ can be obtained after the convolution operation. The pooling operation is often carried out to reduce the dimension of the features extracted by the convolution kernel and prevent the number of features from being too large and the classification model from being too fitted. In this work, maximum pooling is used to process the output of $\mathrm{CNN}$, i.e., taking the maximum value of the second dimension in $O_{1} \in R^{M \times\left(N_{-} S 1+1\right)}, O_{2} \in R^{M \times\left(N_{-} S 2+1\right)}$, and $O_{3} \in R^{M \times\left(N_{-} S 3+1\right)}$ to obtain the vector, which is the result of pooling. As shown in Figure 2, for the convolution kernel $f_{1}$, we take the maximum value in the convolution result $O_{1}$ to obtain the pooled result of a convolution kernel. In the same way, we calculate the results of $f_{1}$ convolution kernels to obtain the $m$-dimensional vector $O_{1}^{\prime} \in R^{M}, O_{2}^{\prime} \in R^{M}$, and $O_{3}^{\prime} \in R^{M}$, which will be used as input for the next phase of the model.

3.2.4. DRL Model. BRCNN model uses the strategic network of DRL to independently extract and classify temporal related words and delete irrelevant words. By predicting the behavior of each word in the sentence, the strategic network determines the deletion or retention behavior of words, i.e., sampling the behavior of each word to achieve the purpose of purifying text information.

In this work, we aim to classify the tenses of a single sentence sample $X$ and produce collocation results. The word vector matrix of $X$ can be expressed as $\left(x_{1}, x_{2}, \ldots, x_{N}\right)^{T}$, where $x_{t}$ corresponds to the vector representation of the th word. The status, behavior, and reward of policy network are introduced as follows. We note that the state of the policy network at time $t$ is $s_{t}(t=1,2, \ldots, N)$. In the BRCNN model, the state $s_{t}(t=1,2, \ldots, N)$ is composed of the word vector input $x_{t}$ of the th word and the hidden state information obtained at time $t$ of the bidirectional cyclic neural network. $a_{t}$

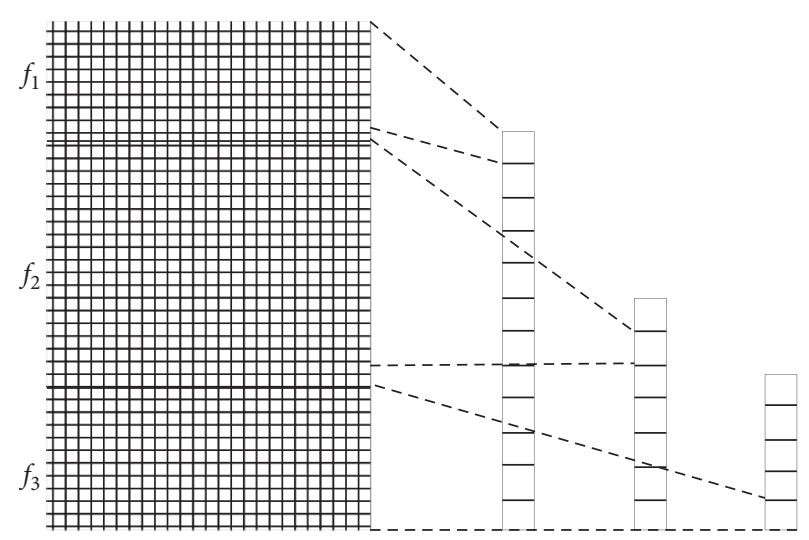

FIgURE 2: Convolution operation of the convolution layer.

$(t=1,2, \ldots, N)$ denotes the behavior of the policy network at time $t$. In the BRCNN model, the goal is to extract words related to tenses, and thus, the behavior $a_{t}$ is set as the deletion or retention of the th word. We set $a_{t} \in\{0,1\}$, where 0 indicates deletion and 1 indicates reservation. During training stage, the strategic network samples the behavior of each word in probability according to the following equation:

$$
\pi\left(\frac{a_{t}}{s_{t} ; \Theta}\right)=\operatorname{Sigmoid}\left(W_{R L} \cdot s_{t}+b_{R L}\right),
$$

where $\pi\left(a_{t} \mid s_{t} ; \Theta\right)$ denotes the probability of selecting behavior $a_{t}$ in state $s_{t}$. The probability calculation of BRCNN model policy network adopts the sigmoid activation function, which can map each input value to the interval from 0 to 1 as the probability of retaining the current word. Then, we subtract this value from 1 to be the probability of word deletion. $\Theta=\left\{W_{R L}, b_{R L}\right\}$ represents the parameters of the BRCNN model policy network. When the policy network is trained and tested, the behavior $a_{t}$ is selected according to (11), where $t=1,2, \ldots, N$.

$$
a_{t}=\arg \max _{a} \pi\left(\frac{a_{t}}{s_{t} ; \Theta}\right) .
$$

The reward value of the strategic network is denoted as $R_{N}$. In the BRCNN model, the behavior is sampled by the strategic network, the forward and backward word order information is extracted by the bidirectional cyclic neural network, and the word-level text is represented and classified by the $\mathrm{CNN}$. The classification result label can be obtained to calculate the reward value $R_{N}$ of the strategic network. $P(y \mid X)$ represents the probability of dividing text $X$ into category $y$, where $y$ represents category information and $X$ is classification text.

$y_{\text {real }}$ denotes the real label of sample $X$, and the probability of correct classification is $P\left(y_{\text {real }} \mid X\right)$. To delete as many independent words as possible and ensure the accuracy of classification, the number of words deleted is considered in the reward value function $R_{N}$. The number of words deleted by the strategic network is $L$, and the number of words deleted by $X$ is $N$, and thus, the definition of reward value function $R_{N}$ is shown as follows: 


$$
R_{N}=\log \left(P\left(y_{\text {real }} \mid X\right)\right)+\lambda \frac{L}{N}
$$

The reward value function $R_{N}$ of the policy network combines the probability of correct classification with the word deletion ratio " $L / N$ " for obtaining a higher probability of correct classification when deleting as many words irrelevant to the classification task as possible. $\lambda$ is a super parameter that balances the two factors of correct classification probability $P\left(y_{\text {real }} \mid X\right)$ and word deletion ratio " $L / N$."

\section{Simulation Experiments}

This section compares and analyzes the experimental results of the word-level text representation and tense collocation model based on DRL. In this section, we introduce the content and source of the experimental dataset, and the setting of parameter in the experiment. All the experiments are conducted on $8 \mathrm{~GB}$ RAM, Intel Core i7 processor with $3.60 \mathrm{GHz}$, and Windows 10 operating system. The method is implemented using the MATLAB R2018b Win64.

4.1. Dataset Collection. There are five basic text classification datasets used in this experiment. The number of categories, the average length of text, the number of samples, the number of thesauri, and the number of samples in the test set are shown in Table 1:

(1) MPQA text dataset: MPQA opinion corpus comes from various news articles and is marked by Wiebe et al., which contain opinions and a series of private states, such as emotion, speculation, and belief

(2) CR text dataset: CR is customer comments on 14 Amazon products, which are divided into positive and negative user emotions

(3) MR text dataset: MR, i.e., movie reviews, is about customers' emotional evaluation of a series of films, including positive and negative emotions

(4) Subj text dataset: subjectivity (Subj) dataset is a subjective and objective emotion classification dataset

(5) TREC text dataset: TREC is a dataset for classifying problems

The initialization word vector in this experiment comes from the trained word vector of Google, i.e., GoogleNewsvectors-negative300.bin, which is trained by the CBOW model on the dataset Google News. Google News contains about 100 billion English words, and the CBOW model finally obtains the vector representation of 3 million words or phrases, in which the dimension of each word vector is 300. For the words that do not appear in the 3-million-word list, the model generates 300 dimensional vectors randomly by means of uniform distribution:

Network weight: the network parameters involved in the BRCNN model are initialized with a normal distribution with a mean value of 0 and a standard deviation of 0.1 , and the bias terms are initialized to 0.1 .
TABLE 1: A summary of the datasets.

\begin{tabular}{lccccc}
\hline Dataset & MPQA & CR & MR & Subj & TREC \\
\hline Number of categories & 2 & 2 & 2 & 2 & 6 \\
Average length & 2.9 & 18.5 & 19.5 & 22.4 & 10.7 \\
Number of samples & 10606 & 3775 & 10662 & 10000 & 5952 \\
Number of thesauri & 6246 & 5340 & 18765 & 21323 & 9592 \\
Number of test samples & 450 & 280 & 360 & 680 & 500 \\
\hline
\end{tabular}

The settings come from the optimal simulation based on different combinations.

Hyperparameters: the Adam method is used to train the model in the experiment, and the learning rate is 0.001 . L2 regularization parameters with the size of 0.0001 are used in the full-connection layer to prevent overfitting. The data input batch is 64 . There are 128 hidden units in the cyclic neural network. The CNN model contains three types of convolution kernels with window sizes of 3, 4, and 5, and 100 convolution kernels are set for each type. $\lambda$ of reward function $R_{N}$ in policy network is 0.1. Similarly, the setting method is to make different combinations of simulation and select the optimal simulation.

4.2. Results and Analysis. The results of comparative analysis of the words with the largest number of deletions or more typical words in the five datasets are shown in Table 2. The number of occurrences represents the total number of occurrences of a word in the dataset, the number of retentions is the number of words retained by the BRCNN model, and the retentions percentage is the ratio of retained words to the number of occurrences.

As can be seen from Table 2, the BRCNN model can effectively retain tense words in characterization data and make accurate tense collocation based on these words. In the CR dataset, "always" has the highest number of occurrences, but the classification accuracy is lower than "before," which shows that the temporal feature of "before" is stronger. In the MPQA dataset, the retention percentage of "normally" is relatively low, indicating that its temporal feature is weak. In MR and Subj datasets, the number of occurrences and retention percentage of "recently" are relatively high, which indicates that it is a typical word with strong temporal characteristics. In the TREC dataset, the number of occurrences of "yet" is low, but the retention percentage is high. This means that although the word is used less, the temporal feature is obvious.

Table 3 shows the accuracy of BRCNN in the English tense collocation proposed in this study. To objectively evaluate the results of temporal collocation, HSASRL [23], SRML [24], and Usr-mtl [25] are introduced as comparison models. It can be seen from the results in Table 3 that the accuracy of BRCNN is higher than HSASRL and Usr-mtl. Compared with the SRML model, the BRCNN model also obtains the highest accuracy in the MPQA, MR, and TREC databases. The reason for experimental results is that BRCNN optimizes the temporal description of sentences using the Allen interval algebra and establishes the 
TABLE 2: BRCNN reserved word.

\begin{tabular}{|c|c|c|c|c|}
\hline Datasets & Words & Number of occurrences & Number of retentions & Retention percentage \\
\hline MPQA & Always & 2228 & 1982 & 88.96 \\
\hline MPQA & Before & 3419 & 3324 & 97.22 \\
\hline MPQA & After & 6751 & 6624 & 98.11 \\
\hline MPQA & Since & 2345 & 2134 & 91.00 \\
\hline MPQA & Now & 12058 & 10859 & 90.05 \\
\hline MPQA & Usually & 6843 & 6029 & 88.56 \\
\hline CR & Always & 79558 & 70020 & 88.01 \\
\hline $\mathrm{CR}$ & Before & 57485 & 56988 & 99.14 \\
\hline $\mathrm{CR}$ & After & 48784 & 41194 & 84.44 \\
\hline CR & Since & 2934 & 2756 & 93.93 \\
\hline $\mathrm{CR}$ & Now & 3050 & 2691 & 88.23 \\
\hline MR & Always & 5331 & 5126 & 96.15 \\
\hline MR & Before & 2569 & 2366 & 92.10 \\
\hline MR & After & 2302 & 2103 & 91.36 \\
\hline $\mathrm{MR}$ & Since & 5037 & 4829 & 95.87 \\
\hline MR & Recently & 8975 & 8690 & 96.82 \\
\hline MR & Comedy & 1843 & 1657 & 89.90 \\
\hline Subj & Before & 1665 & 1423 & 85.47 \\
\hline Subj & After & 5641 & 5564 & 98.63 \\
\hline Subj & Since & 4892 & 4688 & 95.83 \\
\hline Subj & Recently & 9281 & 9024 & 97.23 \\
\hline Subj & Yet & 6574 & 6322 & 96.117 \\
\hline Subj & Ever & 9524 & 9367 & 98.35 \\
\hline TREC & Before & 9661 & 9554 & 98.98 \\
\hline TREC & After & 6541 & 6317 & 96.58 \\
\hline TREC & Since & 5236 & 4594 & 87.74 \\
\hline TREC & Recently & 8563 & 8364 & 97.67 \\
\hline TREC & Yet & 2566 & 2496 & 97.27 \\
\hline TREC & Ever & 9325 & 8864 & 95.05 \\
\hline
\end{tabular}

TABLE 3: Temporal collocation accuracy of comparison models.

\begin{tabular}{lcccc}
\hline Dataset & HSASRL $(\%)$ & SRML $(\%)$ & Usr-mtl $(\%)$ & BRCNN $(\%)$ \\
\hline CR & 88.92 & 92.71 & 83.26 & 91.92 \\
MPQA & 84.51 & 89.44 & 80.86 & 93.76 \\
MR & 85.18 & 87.49 & 89.73 & 92.11 \\
Subj & 83.37 & 94.88 & 90.26 & 87.57 \\
TREC & 85.56 & 90.33 & 88.71 & 95.51 \\
Avg accuracy & 85.51 & 90.97 & 86.56 & 92.17 \\
\hline
\end{tabular}

corresponding relationship between sentences and tenses by combining the high-precision text abstract with the bidirectional cyclic neural network and the convolution neural network. In addition, to further optimize the temporal expression of the text, BRCNN uses the intelligent decision-making ability of RL to effectively retain the tense words and completes the tense collocation with these words.

\section{Conclusions}

In this work, we propose a vector representation, that is, relational vector based on the relational expression of interval in Allen's interval algebra theory, and define the compound and transfer operations of relations on relational vectors, which provide support for the application of relational vectors in temporal reasoning. In addition, based on this temporal representation method, we propose a BRCNN model for temporal word classification and temporal collocation. The model realizes the text expression based on the bidirectional cyclic neural network and the convolutional neural network, and the classification of temporal words using the DRL model. It carries out the corresponding word collocation according to the calculated tenses. Through the experimental comparison of the five models on the datasets, i.e., MPQA, CR, MR, Subj, and TREC, it is proved that the BRCNN proposed in this work performs well in temporal collocation.

As a novel English tense evaluation mechanism, it has some limitations. For example, this study lacks the largescale experiments. In addition, this study has no sufficient theory analysis. In future, we will address the abovementioned limitations. In particular, we plan to make the practical experiments based on the constructed platform.

\section{Data Availability}

The data used to support the findings of this study are available from the corresponding author upon request. 


\section{Conflicts of Interest}

The authors declare that they have no conflicts of interest.

\section{Acknowledgments}

This work was supported by the Heilongjiang Project: Research on Etiquette Discourse Based on Ecological Linguistics, under Grant No. 18YYC263.

\section{References}

[1] Y. Wang and W. Xu, "Leveraging deep learning with LDAbased text analytics to detect automobile insurance fraud," Decision Support Systems, vol. 105, no. 1, pp. 87-95, 2018.

[2] D. S. Kermany, M. Goldbaum, W. Cai et al., "Identifying medical diagnoses and treatable diseases by image-based deep learning," Cell, vol. 172, no. 5, pp. 1122-1131, 2018.

[3] A. Khalil, M. Jarrah, M. Al-Ayyoub, and Y. Jararweh, "Text detection and script identification in natural scene images using deep learning," Computers \& Electrical Engineering, vol. 91, no. C, pp. 107043-107058, 2021.

[4] J. Huan, A. A. Sekh, C. Quek, and D. K. Prasad, "Emotionally charged text classification with deep learning and sentiment semantic," Neural Computing \& Applications, vol. 1, no. 1, pp. 1-11, 2021.

[5] N. Sun and C. Du, "News text classification method and simulation based on the hybrid deep learning model," Complexity, vol. 2021, no. 1, 11 pages, Article ID 8064579, 2021.

[6] H. Klaudel, M. Koutny, Z. Duan, and B. Moszkowski, "From box algebra to interval temporal logic," Fundamenta Informaticae, vol. 167, no. 4, pp. 323-354, 2019.

[7] F. Grandi, F. Mandreoli, R. Martoglia, and W. Penzo, "Unleashing the power of querying streaming data in a temporal database world: a relational algebra approach," Information Systems, vol. 103, no. 13, Article ID 101872, 2021.

[8] J. F. Allen, "Maintaining knowledge about temporal intervals," Communications of the ACM, vol. 26, no. 11, pp. 832-843, 1983.

[9] S. Huang, X. Li, Z. Zhang, F. Wu, and J. Han, "User-ranking video summarization with multi-stage spatio-temporal representation," IEEE Transactions on Image Processing, vol. 28, no. 1, pp. 2654-2664, 2018.

[10] E. Santos, "Cost-based temporal reasoning," Information Sciences, vol. 482, no. 1, pp. 392-418, 2019.

[11] A. Leeuwenberg and M.-F. Moens, "A survey on temporal reasoning for temporal information extraction from text," Journal of Artificial Intelligence Research, vol. 66, pp. 341-380, 2019.

[12] F. Ding, G. Ma, Z. Chen, J. Gao, and P. Li, "Averaged soft actor-critic for deep reinforcement learning," Complexity, vol. 2021, Article ID 6658724, 6 pages, 2021

[13] X.-1. Chen, L. Cao, C.-x. Li, Z.-X. Xu, and J. Lai, "Ensemble network architecture for deep reinforcement learning," Mathematical Problems in Engineering, vol. 2018, Article ID 2129393, 6 pages, 2018.

[14] J. Liu and L. Feng, "Diversity evolutionary policy deep reinforcement learning," Computational Intelligence and Neuroscience, vol. 2021, Article ID 5300189, 11 pages, 2021.

[15] X. Zhang, J. Zhao, and Y. Lecun, "Character-level convolutional networks for text classification," Neural Information Processing Sys, vol. 1, no. 1, p. 1, 2015.

[16] Y. Kim, "Convolutional neural networks for sentence classification," in Proceedings of the Conference on Empirical methods in natural language processing, pp. 1746-1751, Doha, Qatar, October 2014.

[17] T. Mikolov, M. Karafiat, L. Burget, C. Jan, and K. Sanjeev, "Recurrent neural network based language model," in Proceedings of the 11th Annual Conference of the International Speech Communication Association, INTERSPEECH 2010, pp. 1045-1048, Chiba, Japan, September 2010.

[18] S. Lai, L. Xu, K. Liu, and J. Zhao, "Recurrent convolutional neural networks for text classification," Association for the Advancement of Artificial Intelligence, vol. 333, pp. 22672273, 2015.

[19] D. Tang, B. Qin, and T. Liu, "Document modeling with gated recurrent neural network for sentiment classification," in Proceedings of the Conference on Empirical Methods in Natural Language Processing, pp. 1422-1432, Lisbon, Portugal, September 2015.

[20] L. Yu, W. Zhang, J. Wang, and Y. Yu, "SeqGAN: sequence generative adversarial nets with policy gradient," in Proceedings of the National Conference on Artificial Intelligence, pp. 2852-2858, San Francisco, CA, USA, August 2017.

[21] B. Bennett and A. Cohn, "MultiDimensional multi-modal logics as a framework for spatio-temporal reasoning," Applied Intelligence, vol. 1, no. 1, p. 1, 2002.

[22] A. Morales, I. Navarrete, and G. Sciavicco, "Using temporal logic for spatial reasoning: temporalized propositional neighborhood logic," Eurocast, vol. 1, no. 1, pp. 313-320, 2007.

[23] F. Mohsen, J. Wang, and K. Al-Sabahi, "A hierarchical selfattentive neural extractive summarizer via reinforcement learning (HSASRL)," Applied Intelligence, vol. 2, no. 1, p. 1, 2020.

[24] D. Zhao, J. Wang, H. Lin et al., "Sentence representation with manifold learning for biomedical texts," Knowledge-Based Systems, vol. 218, no. 4, pp. 106869-106881, 2021.

[25] W. Xu, S. Li, and Y. Lu, "Usr-mtl: an unsupervised sentence representation learning framework with multi-task learning," Applied Intelligence, vol. 1, no. 1, pp. 1-16, 2020. 